\title{
The ten-channel pulsed radar reflectometer at the TEXTOR-94 tokamak
}

\author{
J. C. van Gorkom, ${ }^{a)}$ M. J. van de Pol, and A. J. H. Donné \\ FOM-Instituut voor Plasmafysica Rijnhuizen, Association Euratom-FOM, Trilateral Euregio Cluster, \\ P.O. Box 1207, NL-3430 BE Nieuwegein, The Netherlands
}

(Presented on 19 June 2000)

\begin{abstract}
A new ten-channel pulsed radar reflectometer has been taken into operation at the Torus Experiment for Technology Oriented Research-94. The system will be used simultaneously as a density profile and as a density fluctuation diagnostic. Ten density layers from $0.4 \times 10^{19}$ to $4 \times 10^{19} \mathrm{~m}^{-3}$ can be probed simultaneously at $2 \mathrm{MHz}$. The design of the diagnostic has undergone some changes, to improve reliability of the system. Examples of time delay measurements are shown. These examples illustrate the fluctuation capabilities of the diagnostic at high time resolution, as well as the response of the different channels to density profile changes. (C) 2001 American Institute of Physics.
\end{abstract}

[DOI: 10.1063/1.1316747]

\section{INTRODUCTION}

Pulsed radar reflectometry is based on a time-of-flight measurement of short $(\sim 1 \mathrm{~ns})$ microwave pulses that are launched into the plasma. The pulses are reflected in the plasma at density layers where the microwave frequency equals the local plasma frequency (in case of ordinary mode polarization). The flight time of the pulses between transmission and detection is measured, and contains the information on the positions of the reflecting layers. ${ }^{1,2}$

The system currently installed at the Torus Experiment for Technology Oriented Research (TEXTOR)-94 is a tenfrequency system running at a $20 \mathrm{MHz}$ total pulse repetition rate. This translates to ten-channel measurements at $2 \mathrm{MHz}$, or four or two channels at a maximum rate of 5 or $10 \mathrm{MHz}$. The accuracy in the time-of-flight determination for a single pulse equals 74 ps. Several subsequent pulses can be averaged to lower this figure further. The diagnostic has a threefold goal: to study density profile developments, coherent macroscopic fluctuations, and broadband microscopic fluctuations.

Plans for the present pulsed radar system have been described in some detail in this journal two years ago. ${ }^{3}$ The present article will concentrate on changes that had to be made in the design, as well as on first measurements, showing some of the capabilities of the diagnostic. A brief description of the system, detailing only the new additions, is followed by a discussion on the reasons for and on the consequences of some of the design changes. In the last section experimental results on magnetohydrodynamic (MHD) fluctuations, as well as the very first profile measurements over the full accessible density range, are presented.

\section{TECHNICAL DESCRIPTION}

The experimental layout is illustrated in Figs. 1 and 2 for the $K$ and for the $K a$ band system, respectively (the $U$ band system being very similar to the $K a$ band system). Ten Impatt oscillators are used as microwave sources. Each of them

${ }^{\text {a)} E l e c t r o n i c ~ m a i l: ~ g o r k o m @ ~ @ r i j n h . n l ~}$ is equipped with an individual fast pulse-forming modulator, consisting of a circulator and a varactor diode in a tuned cavity. These modulators can reach very high on-off ratios of up to $50 \mathrm{~dB}$ in lab tests, but need a carefully tuned bias voltage to ensure maximum isolation in the off state.

After the modulators, the ten different microwave frequencies are merged into three waveguide systems by combiners, consisting of resonant cavities and circulators. Mainly oversized waveguide is used to cover the $10 \mathrm{~m}$ distance to the antenna system, in order to minimize losses and pulse broadening. For each waveguide band, a separate transmitter and receiver horn antenna is used. A short distance from the antennas, a small part of the transmitted pulse is coupled directly into the receiver waveguide via a bypass loop, consisting of two $10 \mathrm{~dB}$ directional couplers, a variable attenuator, and a short section of waveguide. The pulses traveling via the bypass loop function as a start pulse for the time-offlight measurement, independent of possible jitter in the electronics.

Also shown in Fig. 2 is one of two tunable sources, which can be swept in frequency over the indicated range

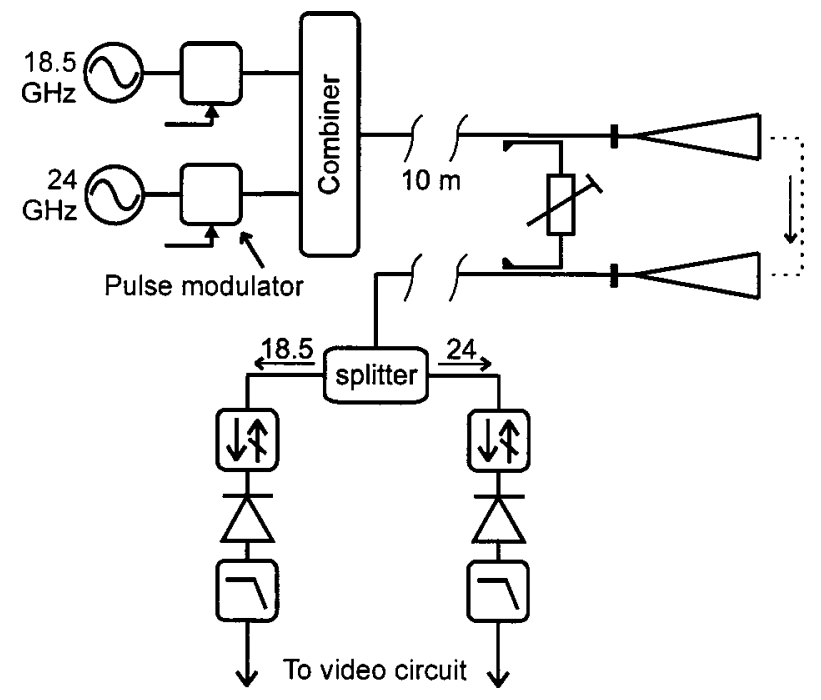

FIG. 1. Schematic layout of the $K$ band reflectometer. 


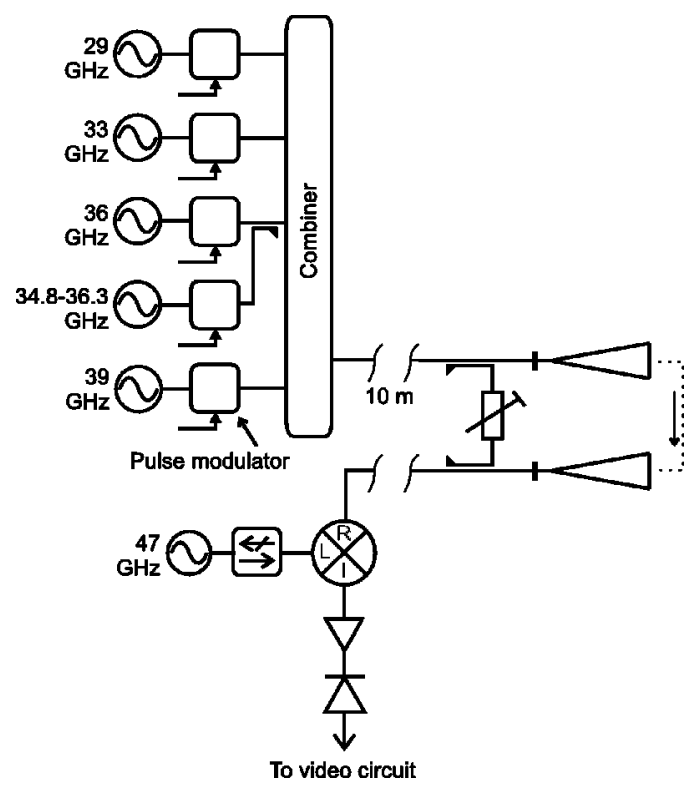

FIG. 2. Schematic layout of the $K a$ band reflectometer. The $U$ band system is similar, with the fixed frequencies $47,51,54$, and $57 \mathrm{GHz}$, a tunable source around $51 \mathrm{GHz}$, and a local oscillator at $39 \mathrm{GHz}$.

during a discharge by means of arbitrary wave generators. These sources have not been commissioned yet.

The receiver for the $K$ band channels is based on homodyne detection, after a frequency selective splitter. For the $K a$ and $U$ band systems heterodyne detection is used. In the original design, four different local oscillators (LOs) per receiver were switched on sequentially. These LOs matched to the probing frequencies such that the resulting intermediate frequency (IF) was always $18 \mathrm{GHz}$. Thus a narrowband receiver could be constructed. This scheme has been abandoned in favor of broadband heterodyne receivers. Gunn oscillators at 47 and $39 \mathrm{GHz}$ are used as LO, in combination with broadband $(8-18 \mathrm{GHz})$ IF amplifiers and tunnel diode detectors. Filter banks will be installed shortly, to ensure frequency selectivity.

After detection and video amplification, time pickoff is achieved by means of constant fraction discriminators, one for each waveguide band (originally, only one discriminator was available for all bands combined). These discriminators eliminate the influence of amplitude variations in the detected pulses on the time delay measurement. The time delay between the pairs of pulses (from the bypass waveguide and from the plasma reflection) is then measured by a custombuilt counter system, consisting of eight parallel gated counters.

\section{DESIGN CHANGES}

Since the very first measurements with part of the present system in June 1998, its performance has continuously been monitored and improved. Many minor problems have been solved, but also some more major changes have been made. Those changes will be discussed in this section.

Probably the most persistent problems stem from the fact that the varactor modulators have narrowband isolation performance, and that their isolation value is very sensitive to the applied bias voltage. The pulse drivers used for control-

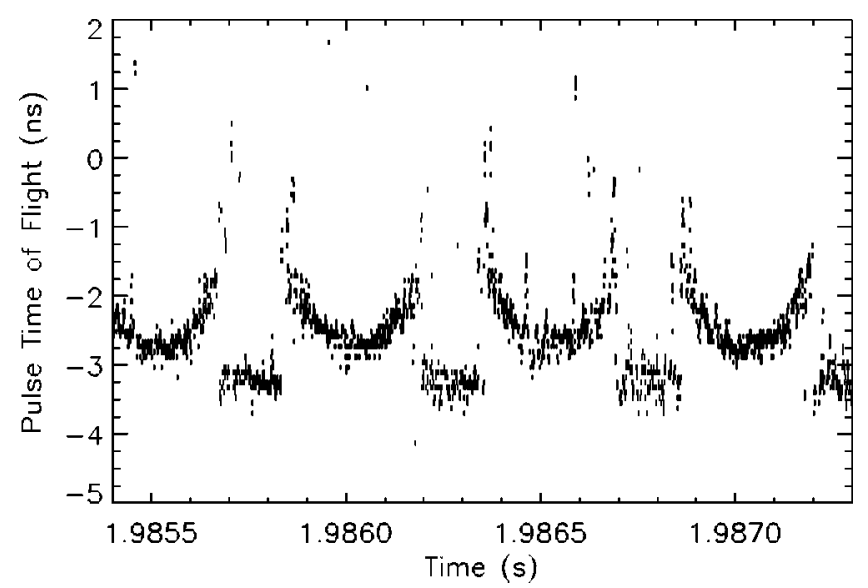

FIG. 3. A magnetic island observed by the $36 \mathrm{GHz}$ channel. No filtering has been applied: each dot represents one reflected pulse. The time of flight for reflection off the back wall of the vessel in vacuum is defined to be zero.

ling the modulators proved to be not stable enough in $\mathrm{dc}$ level to maintain optimum isolation in the modulators over time. Moreover, the isolation depends on the frequency stability of the oscillators. The original experimental layout is particularly sensitive to these problems, since the oscillators play a double role: as rf source for the one band, as LO source for the other band. Any modulator leakage thus influences both heterodyne receivers: one as a continuous rf signal (modulated by plasma fluctuations, interfering with the bypass signal), and the other as continuous LO power at an undesired frequency. Because improving and stabilizing the pulse driver units was not a practical option on the short term, the decision was made to move away from the switched-LO receiver configuration, at least for the first campaigns. Instead the two broadband heterodyne receivers were installed. Since this change, reliable operation has been achieved. Modulator leakage occasionally still causes problems, but usually influences only a single channel.

There is a drawback to this improvement, which lies mainly in the broad bandwidth of the new receivers. This means that more noise from the plasma (mainly electron cyclotron emission radiation) is entering the system. Whereas the instantaneous bandwidth of the switched receivers could be reduced to less than $2 \mathrm{GHz}$, the broadband receivers have a bandwidth of $10 \mathrm{GHz}$. The filter banks will reduce this to about $4 \mathrm{GHz}$ (1 GHz per channel). Two factors alleviate the effect of the increase in noise bandwidth somewhat: first, higher signal powers are available, since the oscillators no longer have to generate LO power. Second, each waveguide band receiver has been equipped with its own constant fraction discriminator, so that noise in the different bands no longer adds up. This also gives the possibility of tuning the pulse height detection threshold per band. The last asset of the switched-LO scheme, time window selection, has been lost, but in the TEXTOR case of bistatic detection this is no great concern, since no vacuum break reflection occurs before the plasma reflection. Any time window selection necessary to eliminate multiple reflections can be done in software.

Other changes to the system include a change of the bypass location. This waveguide loop has been moved some- 


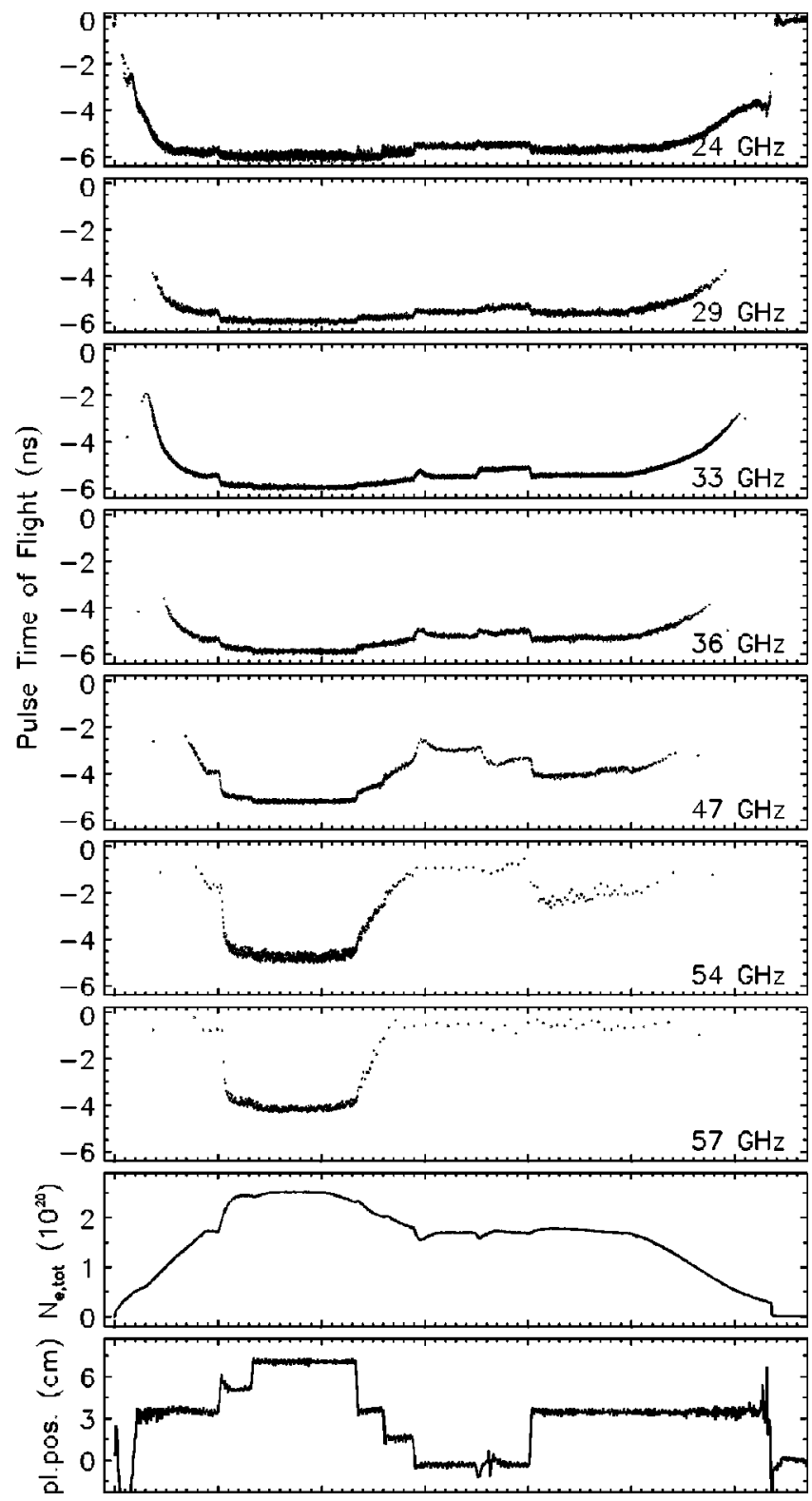

FIG. 4. Pulsed radar traces for TEXTOR discharge 88027. The time of flight for reflection off the back wall of the vessel in vacuum is defined to be zero. Filtering has been applied: each dot represents an average over 256 reflected pulses. Due to a malfunction, the counter system accuracy was eight times lower than normal, so this pulse averaging is equivalent to averaging four pulses at normal accuracy. In the lowest two graphs, the total number of electrons $N_{e, \text { tot }}$ and the horizontal plasma position as measured by a ninechannel interferometer are plotted.

what further away from the vessel, since the shortest time delay detectable by the constant fraction discriminator turned out to be about $8 \mathrm{~ns}$, instead of $4 \mathrm{~ns}$ as tests seemed to indicate, for the pulses we produce. A consequence of this change is that we lose measuring range at the top of the time delay range, so that delayometry (in low-density plasmas, where pulses are not reflected by the plasma but by the back wall of the vessel) now has become largely impossible unless an adaptation to the counter system is be made. This is no great loss, however, since signal levels for the back wall reflection on TEXTOR are anyway only marginally detectable, and since, at typical TEXTOR densities, most or all of the channels reflect in the plasma.

\section{EXPERIMENTAL RESULTS}

In the course of the installation and commissioning of the different parts of the system, physics experiments have already been done. A physics campaign on MHD perturbations has been undertaken, using until now mainly the $K$ and $K a$ band channels. The first full density profile measurements have been obtained recently, following the final commissioning of the $U$ band receiver. The fluctuation studies will benefit much from the addition of the tunable sources. These will enable radial correlation measurements of the turbulence, as well as detailed scans over, e.g., MHD perturbations.

The capabilities of the reflectometer as a fluctuation diagnostic are illustrated in Fig. 3. This trace illustrates a phenomenon that always occurs when large magnetic islands are present in the TEXTOR plasma: whenever a channel is probing in the region of the island, discontinuities in the signal occur in between the $\mathrm{O}$ and the $\mathrm{X}$ point location of the island. This is connected with the presence of a secondary reflecting layer inside the island, ${ }^{4}$ with the pulses reflecting alternately from inside the island and from the density layer behind the island. An extra confirmation of this density peaking in the islands has recently been obtained by Thomson scattering. ${ }^{5}$

In June 2000, the $U$ band system became fully operational, bringing the total number of channels up to ten. This opens the way for detailed studies of density profile developments. One of the first measurements is shown in Fig. 4. In this discharge, the horizontal position of the plasma was changed in several steps. All reflectometer channels can be seen to react to the position and density changes (the plasma position and the total number of electrons in the plasma have been plotted for comparison). Due to a malfunction in the counter system, the accuracy of the time-of-flight measurement was in this case a factor of 8 less than the normal 74 ps. This has been compensated for by applying a factor of $8^{2}$ extra pulse averaging, at a corresponding loss of time resolution. The higher frequency channels are probing the density profile near its top, according to the interferometer measurements. This explains the low density of detected pulses as the density drops around $2.5 \mathrm{~s}$ : a small and highly curved flux surface far away from the antennas, together with possibly strong pulse broadening due to the shallow density gradient near the top of the profile, causes low reflected pulse amplitudes. In the nearest future Abel inversion techniques will be applied to these measurements, in order to reconstruct the density profile.

\section{ACKNOWLEDGMENTS}

The work described in this article was performed as part of the research program of the Stichting voor Fundamenteel Onderzoek der Materie (FOM), with financial support from the Nederlandse Organisatie voor Wetenschappelijk Onderzoek (NWO) and EURATOM. 
${ }^{1}$ C. A. J. Hugenholtz and S. H. Heijnen, Rev. Sci. Instrum. 62, 1100 (1991).

${ }^{2}$ V. F. Shevchenko, A. A. Petrov, V. G. Petrov, and U. A. Chaplygin, Proceedings of the 20th Conference on Controlled Fusion and Plasma Physics, Lisbon, 1993, Europhysics Conference Abstracts, Vol. 17C, Part III, p. 1167.

${ }^{3}$ C. A. J. Hugenholtz, A. J. H. Donné, B. S. Q. Elzendoom, J. C. van Gorkom, W. Kooijman, H. A. van der Laan, M. J. van de Pol, A. J. Putter,
H. J. F. van Ramele, D. Smit, P. C. de Vries, F. Wijnoltz, W. Pysik, G. Waidmann, and G. P. Ermak, Rev. Sci. Instrum. 70, 1034 (1999).

${ }^{4}$ P. C. de Vries, A. J. H. Donné, S. H. Heijnen, C. A. J. Hugenholtz, A. Krämer-Flecken, F. C. Schüller, and G. Waidmann, Nucl. Fusion 37, 1641 (1997).

${ }^{5}$ C. J. Barth, H. J. van der Meiden, T. Oyevaar, and N. J. Lopes Cardozo, Rev. Sci. Instrum. (these proceedings). 